\title{
PROOF OF CONCEPT OF A NEW GLUCOSE SENSING TECHNOLOGY: COLOR-CHANGING HYDROGELS INCLUDING AU NANOPARTICLES
}

\author{
Esch, C. ${ }^{1,3}$, Galperin, A. ${ }^{1}$, Krolitzki, B. ${ }^{3}$, Glasmacher, B. ${ }^{3}$, Shen, A. ${ }^{2}$ and Ratner, B.D. ${ }^{1}$ \\ ${ }^{1}$ Department of Bioengineering, University of Washington, USA \\ ${ }^{2}$ Department of Mechanical Engineering, University of Washington, USA \\ ${ }^{3}$ Institute for Multiphase Processes, Leibniz University of Hannover, Germany \\ christina.esch@t-online.de
}

\begin{abstract}
This Master thesis provides a proof of concept for a novel, implantable continuous glucose sensing technology. Immobilized glucose oxidase in a poly 2-hydroxyethyl methacrylate hydrogel is used to enzymatically convert an increase in glucose level to a local decrease in $\mathrm{pH}$, which leads to a swelling of the hydrogel. Encapsulated gold nanoparticles in the gel allow an optical readout of the glucose concentration. Experimental methods include hydrogel synthesis, nanoparticle encapsulation and finally glucose sensing with the hydrogel by UV-vis measurements. Two possible readouts, absorbance change and frequency shift, are discussed and tested. While a frequency shift is not detected, results show that the absorbance of the gel is proportional to glucose level, making it a promising concept for continuous glucose monitoring.
\end{abstract}

Keywords: Glucose sensor, Au nanoparticles, hydrogel synthesis, enzyme immobilization, porous scaffolds

\section{Introduction}

Diabetes mellitus is a disease where high blood sugar levels cannot be regulated automatically to physiological values from the body. Approximately $8.6 \%$ of the Eurpoeans are currently affected of diabetes. In 2030, this number will rise up to $10 \%$, [1]. The increase in diabetes patients issues a challenge to researchers and physicians. The common goal is to develop implantable, compact and accurate systems which continuously measure the glucose level. This Master thesis develops a new concept based on an enzyme sensor. The sensor is made of a $\mathrm{pH}$ sensitive poly 2-hydroxyethyl methacrylate (pHEMA) hydrogel where the enzyme glucose oxidase (GOx) and $14 \mathrm{~nm}$ gold ( $\mathrm{Au}$ ) nanoparticles are encapsulated. Once the hydrogel is exposed to physiological $(65-180 \mathrm{mg} / \mathrm{dl})$ and pathological $(>250 \mathrm{mg} / \mathrm{dl})$ glucose levels, the GOx catalyzes degradation of glucose to gluconic acid, [2]. This reaction leads to a decrease in $\mathrm{pH}$ level within the hydrogel. The $\mathrm{pH}$ sensitive component of the hydrogel, poly N,N-dimethylaminoethyl methacrylate ( $\mathrm{pN}$ NDMAEM), reacts to the decrease in $\mathrm{pH}$ level by intensive swelling. The presence of the encapsulated Au nanoparticles in the hydrogel translates the swelling into a measurable value. The swelling leads to an increase of the distance between each nanoparticle. The absorption and reflection of the incident light change and will cause a change in intensity of transmitted light.

\section{Methods}

First, $\mathrm{pH}$-sensitive hydrogels are synthesized. The materials are the two monomers HEMA and the pH-sensitive NNDMAEM, the crosslinker tetraethylene glycol dimethacrylate (TEGDMA, $2 \% \mathrm{~mol} / \mathrm{mol}$ monomer), the UV initiator Irgacure $651(0.25 \% \mathrm{~mol} / \mathrm{mol}$ monomer $)$ and the solvent components ethylene glycol (EG) and water (ratio of $\mathrm{EG} / \mathrm{water}=1: 9$ and 3:7), [3]. UV polymerization time is six minutes. Afterwards, the hydrogels are washed for two days to extract impurities and residues of unreacted monomer reagents to obtain a transparent hydrogel.

Five hydrogels with different molar ratios of HEMA and NNDMAEM are synthesized. Their molar mass ratios are shown in Tab. 1. The synthesized hydrogel samples are stored in buffer solutions $\mathrm{pH}=4,5.5,7.4,8.5$, and 10 for two days.

Table 1: Five hydrogel types with different molar ratios of HEMA and NNDMAEM.

\begin{tabular}{lcc}
\hline \hline Abbreviation & $\begin{array}{c}\text { HEMA } \\
\text { mol [\%] }\end{array}$ & $\begin{array}{c}\text { NNDMAEM } \\
\text { mol [\%] }\end{array}$ \\
\hline \hline HEMA 100 \% & 100 & 0 \\
\hline HEMA 93 \% & 93 & 7 \\
\hline HEMA 86 \% & 86 & 14 \\
\hline HEMA 72 \% & 72 & 28 \\
\hline HEMA 50 \% & 50 & 50 \\
\hline
\end{tabular}

The water content in the hydrogels is calculated with Eq. 1, where $m_{w}$ represents the weight of the wet sample and $m_{d}$ the weight of the dry sample, [3].

$$
\% \mathrm{H}_{2} \mathrm{O}=\frac{m_{w}-m_{d}}{m_{w}} \cdot 100[\%]
$$

$14 \mathrm{~nm}$ sized Au nanoparticles are prepared with the method of TURKEVICH ET AL. $0.01 \%$ w/v concentrated chloroauric acid $\left(\mathrm{HAuCl}_{4}\right)$ is heated to $100{ }^{\circ} \mathrm{C} .1 \% \mathrm{w} / \mathrm{v}$ concentrated sodium citrate ( $2.5 \%$ of the total $\mathrm{HAuCl}_{4}$ volume) are dissolved in the heated $\mathrm{HAuCl}_{4}$ solution, [4]. The resulting particle size is measured with a scanning electron microscope and averages $14 \pm 5 \mathrm{~nm}$.

Au nanoparticles are centrifuged (14,000 rpm for 15 minutes) and added to the monomer solution. The final Au concentration in the hydrogel is $214 \mathrm{ppm}$. To analyze the 
morphology of the entrapped Au nanoparticles in the hydrogels, transmission electron microscope (TEM) measurements are performed. The used device is the Philips CM100 TEM with an acceleration voltage of $100 \mathrm{kV}$.

The next step is to include the enzyme GOx in the hydrogel with the encapsulated Au nanoparticles. The final GOx concentration in the hydrogel is $0.05 \mathrm{mM}$. The glucose sensitive hydrogels with encapsulated Au nanoparticles are stored in physiological and pathological glucose concentrations from 0 to $500 \mathrm{mg} / \mathrm{dl}$ for two days. UV-vis spectra of each hydrogel from 400 to $700 \mathrm{~nm}$ are obtained with the UV-vis Plate Reader Safire2 Tecan.

\section{Results}

Fig. 1 represents the swelling ability of the five hydrogel components in various buffer solutions from $\mathrm{pH}=4$ to 10 .

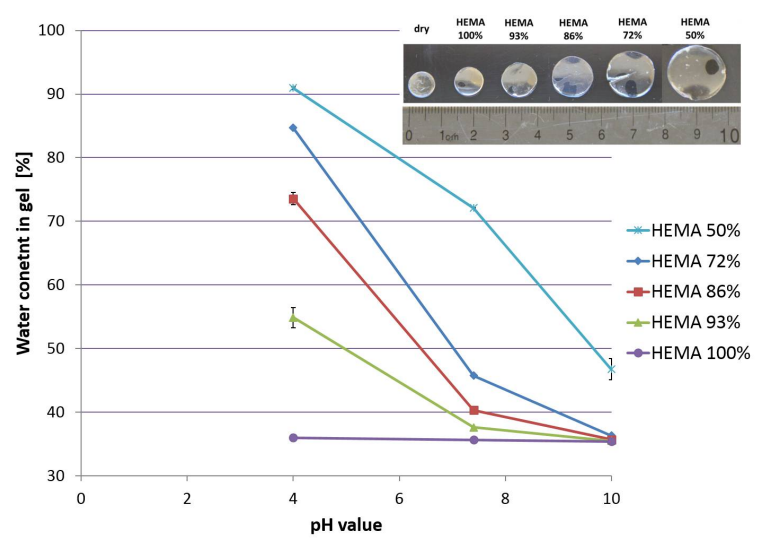

Figure 1: Swelling of the five hydrogel types in $\mathrm{pH}=4,7.4$ and $10(n=3)$ and picture of swollen hydrogels in $\mathrm{pH}=4$

It can be seen that the HEMA $100 \%$ hydrogel without any $\mathrm{pH}$ sensitive polymer pNNDMAEM does not change its swelling behavior in different buffer solutions. However, the swelling ability of the hydrogels rises with the increase in the pNNDMAEM fraction in the hydrogel's composition. This behavior is due to the presence of $\mathrm{pH}$ responsive pNNDMAEM fraction that contains tertiary amino groups. The tertiary amino is protonated and triggers the increase in swelling of the hydrogel's network, [3].

To achieve the biggest change in distance between the encapsulated $\mathrm{Au}$ nanoparticles in the hydrogel and to see a change in absorption, hydrogel type HEMA $72 \%$ is used for further experiments. It shows the highest swelling between physiological $\mathrm{pH}=7.4$ and $\mathrm{pH}=4$.

The encapsulated Au nanoparticles in the hydrogel can be seen on the TEM picture in Fig. 2, a.

The final step is to sense different glucose levels. Results show that higher glucose concentration leads to a swelling of the hydrogels with encapsulated GOx and Au nanoparticles, presented in Fig. 2, b. At the same time the absorbance intensity of the Au nanoparticles decreases. This abatement is shown in Fig. 3, where the absorbance maxima of the hydrogels decreases at a constant wavelength of $531 \mathrm{~nm}$ while the glucose level increases.
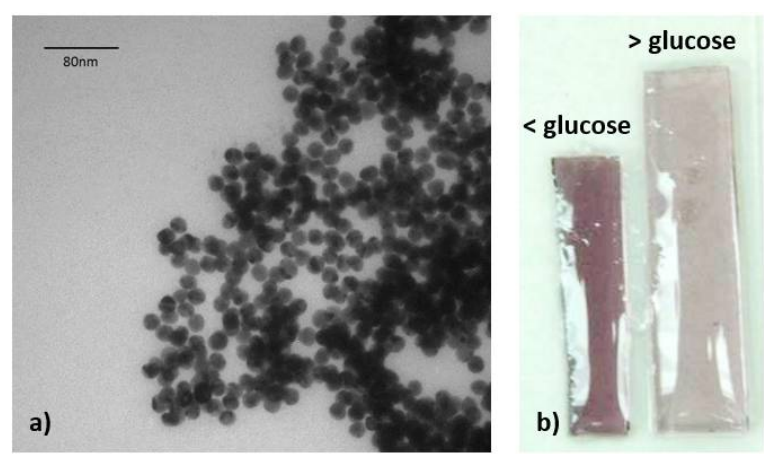

Figure 2: a) TEM of hydrogel including Au nanoparticles (214 ppm); b) swollen hydrogels in low (left) and high (right) glucose concentrations (214 ppm)

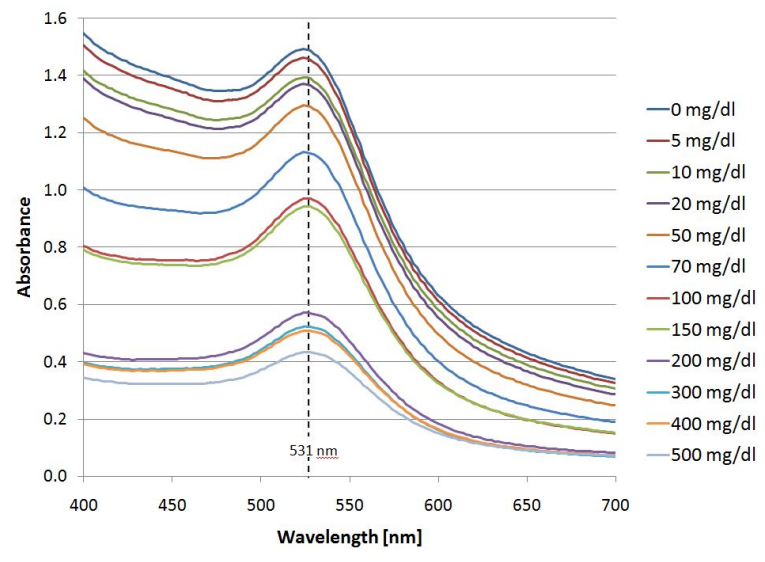

Figure 3: Absorbance scans of samples in different glucose concentrations from 0 to $500 \mathrm{mg} / \mathrm{dl}(\mathrm{n}=6)$

\section{Discussion}

Glucose sensing with pHEMA hydrogels including GOx and $\mathrm{Au}$ nanoparticles is successfully proven to be a viable and effective method for the use in a novel implantable wireless device. The use of optical properties provides a transdermal readout. Future challenges will include the implementation of this technology into an implantable sensor. One possible idea is the inclusion of microdroplets out of the developed hydrogel into a porous scaffold material.

\section{Bibliography}

[1] D. Z. für Diabetesforschung e.V. http://www.dzd-ev.de, 2013-01-29.

[2] D. Matthews, Diabetes, The Facts. Oxford University Press, 1st ed., 2008.

[3] J. Kost et al., "Glucose-sensetive membranes containing glucose oxidase - activity, swelling, and permeability studies," Journal of Biomedical Materials Research, vol. 19, pp. 1117-1133, 1985.

[4] J. Turkevich et al., "Controlled nucleation for the regulation of the particle size in monodisperse gold suspensions," Nature Physical Science, vol. 241, pp. 20-22, 1973. 